\title{
An evaluation of touch-screen input for a HyperCard-based digit-span task
}

\author{
THOMAS A. MARTIN and WILLIAM E. ALLAN \\ Susquehanna University, Selinsgrove, Pennsylvania
}

\begin{abstract}
The touch screen appears to be an ideal input device for human subjects in many research applications in psychology. Nonetheless, the research on relative merits of input devices is inconclusive. The present experiments were carried out to compare touch-screen, mouse, and voice responses on a HyperCard-based digit-span task. The results indicated that touch-screen input has no advantage in comparison to the use of a mouse, but that it produces better performance than voice response does. The latter finding, however, may be an artifact of visual cues embedded in this implementation of the digit-span task.
\end{abstract}

Researchers interested in using computers as devices for stimulus presentation and data acquisition have generally been concerned with finding the simplest, most natural subject-computer interface. This interest is driven by the desire to realize the benefits of computing without forcing subjects into response formats that compromise the study. Keyboard input has been abandoned in favor of game controllers, joy sticks, light pens, mouse devices, touch screens, and interfaces with standard laboratory equipment.

The technology for manufacturing touch-sensitive screens has been available for 25 years, but they have been slow to gain popularity, owing to their expense and to the limitations of touch-sensing technologies (Pickering, 1986). The advantage of a touch-screen system is that it can detect pointing, a highly natural and automatic response. As a consequence, such systems have generated increasing interest in their utility as research tools. Touch screens have been used to administer tests to psychiatric patients (French \& Beaumont, 1987), to perform cognitive assessments of elderly individuals (Carr, Woods, \& Moore, 1986), to study preference and performance in the making of selections in menu-driven software (Karat, McDonald, \& Anderson, 1986), and as a response device in pigeon laboratories (Morrison \& Brown, 1990).

When touch screens have been compared with other input devices, the results have generally supported the utility of touch screens. Karat et al. (1986) found that the touch screen and keyboard surpassed the mouse for menu selection tasks; Carr et al. (1986) concluded that the touch screen is the optimal input device for use by individuals affected by dementia; and French and Beaumont (1987) determined that psychiatric patients prefer the touch screen to the keyboard when completing simple tests. However,

\footnotetext{
We thank Chris Neff for his assistance with this project. Correspondence should be addressed to Thomas Martin, Department of Psychology, Susquehanna University, Selinsgrove, PA 17870.
}

all of these researchers have noted the limitations of touch screens and of computerized testing in general. Karat et al. (1986) found that the keyboard was the preferred selection device when a menu was embedded in a typing task; Carr et al. (1986) commented that some subjects with arthritis had difficulty with the touch screen; and French and Beaumont (1987) determined that psychiatric patients favored manual rather than computerized administration of more complex tasks.

The following studies were precipitated by the first author's observation that many undergraduate subjects in his laboratory seemed rather awkward and uncomfortable when maneuvering a mouse. At that time, no mouseequipped computers were available on the campus for general student use, so few students were experienced with the mouse as a pointing device. It was his concern that discomfort with the mouse might impair subjects' performance on certain types of tasks. Since a touch screen forms a simpler, more natural interface between the user and computer, it seemed reasonable to suppose that the touch screen might allow subjects to perform at their optimal levels.

It also seemed reasonable to hypothesize that impairment caused by difficulty in using a mouse might manifest itself in short-term memory tasks, because short-term memory is known to be volatile and easily affected by interference and anxiety. The digit-span task, in which respondents are asked to repeat series of numbers forward and backward, is one common short-term memory task. It is frequently used in clinical assessment of memory, and it is incorporated in the Wechsler Adult Intelligence Scale-Revised (Wechsler, 1981). Performance on this task is noted for its sensitivity to distraction and anxiety (Anastasi, 1988; Cohen, Montague, Nathanson, \& Swerdlick, 1988).

Experiment 1 was designed to evaluate the effects of mouse versus touch-screen responses; it was hypothesized that subjects would exhibit better performance in the touch-screen condition because of the greater ease of use of the device. 


\section{EXPERIMENT 1}

\section{Method}

Subjects. Twenty-six undergraduates at Susquehanna University participated in the study for extra credit in their introductory psychology course. Of the subjects, 23 were female and 3 were male.

Apparatus. A digit-span task was developed for presentation on a Macintosh SE equipped with IMB of RAM, two internal 800K floppy disk drives, a CMS SD43 external hard drive, and an internally mounted Mac 'n Touch touch screen from MicroTouch Systems. The scripts that controlled the presentation of stimuli and the collection of data were written in HyperTalk, the scripting language of the HyperCard development environment (Apple Computer, 1988). Auditory stimuli were digitized with a Farallon MacRecorder and presented via the internal speaker of the computer. At the time of testing, the software was resident on the hard drive, the touchscreen driver was set to drag mode, and the speaker volume was set to 3 on the Macintosh control panel.

Procedure. The subjects were instructed in the use of either the touch screen or the mouse, then presented with a practice trial. A numeric keypad was displayed on the screen (see Figure 1), and a random series of three digits was presented by highlighting the keys. As each button was highlighted, the number was pronounced by a digitized female voice. When stimulus presentation was finished, the subject was instructed to enter the same series by touching the keypad displayed on the screen or clicking its buttons with the mouse. They were allowed to practice and ask whatever questions they wished; then, the session was begun.

At the beginning of each trial, the keypad was masked, and a field with the message "Touch me when you are ready" (or "Click me when you are ready" for the mouse condition) was displayed on the screen. When the subject touched the screen or clicked the mouse, the keypad

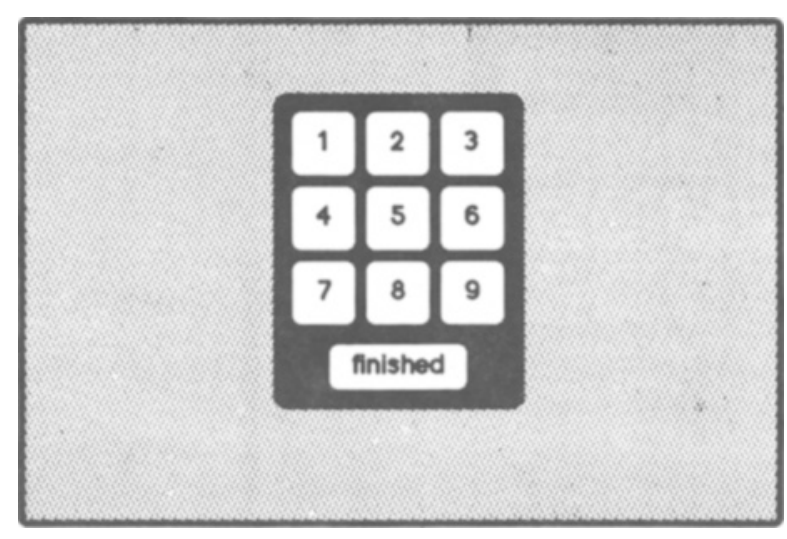

Figure 1. Keypad display. was unmasked, and stimulus presentation was initiated. The first experimental trial presented a randomly generated series of three digits; the length of the stimulus series was increased by one, following each correct response. The forward condition was terminated when the subjects made three consecutive errors or were able to correctly complete a series of nine digits.

When the subjects had completed the digits-forward condition, they were instructed to perform the task again but to answer in reverse order. Another practice trial was presented; then, the subjects were administered the digitsbackward condition. With the exception of the reverse order of response, the backward condition was identical to the forward condition.

After the subjects had completed the forward and backward conditions with one input device, they were instructed in the use of the other device and tested again. The order of device presentation was counterbalanced to control for practice effects. The responses of each subject were stored on data cards in the HyperCard stack that controlled all of the other elements of the experiment. The dependent variable for each condition was the number of digits in the longest series correctly executed.

\section{Results}

A $2 \times 2$ repeated measures analysis of variance revealed a significant effect of direction of response $[F(1,25)$ $=14.00, p<.001]$. Performance in the forward condition $(M=7.14)$ exceeded that in the backward condition $(M=6.27)$. However, the trend toward better performance with the touch screen $(M=6.89)$ as opposed to the mouse $(M=6.51)$ was not significant. No significant interactions were observed.

\section{Discussion}

Contrary to expectation, the touch screen had no discernible advantage over the mouse. Many subjects commented that they enjoyed using the touch screen, but this may have been a function of its novelty as much as of its ease of use. One wonders, however, whether the simplicity of the touch screen might make a significant difference among other populations. It is conceivable that the mouse may be more difficult for young children, persons with developmental disabilities, or older adults than it was for the undergraduates who participated in this study.

The effect of response direction encountered in this study was not surprising; to repeat any sequence in reverse order is typically more difficult than simply to reproduce it. However, the finding does lend support to the validity of this computerized version of the digit-span task by replicating the pattern of response that is expected in the standard form of the task.

The second study was intended to determine whether verbal responses to the HyperCard digit-span task would enhance performance relative to motor responses. Since a verbal reply to aural stimuli may be easier and more 
natural than any type of motor response, it was hypothesized that verbal responses would result in better performance than would the use of the touch screen.

\section{EXPERIMENT 2}

\section{Method}

Subjects. The subjects were 20 undergraduates enrolled in an introductory psychology course who were awarded extra credit for taking part in the study. Eighteen participants were female; 2 were male.

Apparatus. The apparatus for Experiment 2 was identical to that in Experiment 1, except that slight modifications to the HyperCard stack allowed the experimenter to code the accuracy of the subjects' responses during the verbal condition.

Procedure. The touch-screen condition was identical to that in Experiment 1. In the verbal condition, the subjects heard a series of digits pronounced by the computer, but they did not view the screen. They then responded vocally, and the experimenter clicked a button in a dialogue box to indicate whether the subject had repeated the sequence successfully. This procedure closely approximates the standard form of presentation of the digit-span task, in which the examiner says a series of digits and the subject repeats the series. As in the first study, forward and backward conditions were administered within each response condition, response conditions were counterbalanced to control for practice effects, and the dependent variable was the longest series of digits correctly completed in each condition.

\section{Results}

A $2 \times 2$ repeated measures ANOVA indicated that touch-screen responses resulted in better performance than verbal responses did $[F(1,19)=13.10, p<.002]$. Performance in the forward condition exceeded that in the backward condition $[F(1,19)=42.75, p<.001]$. A significant interaction of response format and direction was also present $[F(1,19)=5.52, p<.05]$. Means for each condition are reported in Table 1.

\section{Discussion}

This outcome contradicts the hypothesis and appears to challenge the findings of Beaumont (1985), who reported that touch-screen input on a computerized digitspan task resulted in performance decrements, in comparison with standard verbal presentation and response. However, his procedure involved the visual presentation of stimuli in the touch-screen condition and aural presentation of the stimuli in the verbal response condition. In
Table 1

Mean Digit Span as a Function of Response and Direction

\begin{tabular}{lcc}
\hline & \multicolumn{2}{c}{ Direction } \\
\cline { 2 - 3 } Response & Forward & Backward \\
\hline Touch & 7.75 & 6.70 \\
Verbal & 7.20 & 5.25 \\
\hline
\end{tabular}

the present study, both the visual and the aural stimuli were presented in the touch-screen condition, but only aural stimuli were presented in the verbal response condition. It may be that Beaumont's results reflect the superiority of auditory encoding over visual encoding for performing a digit-span task, and that the present study demonstrates the superiority of combined encoding over purely auditory encoding of series of digits.

\section{GENERAL DISCUSSION}

The touch screen functioned well as a response device for a digit-span task developed in the HyperCard environment, but it showed no clear advantage over other forms of input. Further research is needed to remove the stimulus presentation confound present in the second experiment and fully resolve the question of the touch screen's comparability to verbal response in this task.

\section{REFERENCES}

AnAstasi, A. (1988). Psychological testing. New York: Macmillan. APPLE COMPUTER. (1988). Apple HyperCard script language guide: The HyperTalk language. Reading, MA: Addison-Wesley.

Benumont, J. G. (1985). The effect of microcomputer presentation and response medium on digit span performance. International Journal of Man-Machine Studies, 22, 11-18.

CARR, A. C., WoOds, R. T., Moore, B. J. (1986). Automated assessment of elderly patients: A comparison of two types of response devices. British Journal of Clinical Psychology, 25, 305-306.

Cohen, R. J., Montague, P., Nathanson, L. S., Swerduck, M. E. (1988). Psychological testing: An introduction to tests and measurement. Mountain View, CA: Mayfield.

French, C. C., \& BeAumont, J. G. (1987). The reaction of psychiatric patients to computerized assessment. British Journal of Clinical Psychology, 26, 267-278.

Karat, J., McDonald, J. E., \& Anderson, M. (1986). A comparison of menu selection techniques: Touch panel, mouse and keyboard. International Joumal of Man-Machine Studies, 25, 73-88.

Morrison, S. K., Brown, M. F. (1990). The touch screen in the pigeon laboratory: An initial evaluation of its utility. Behavior Research Methods, Instruments, \& Computers, 22, 123-126.

Pickering, J. A. (1986). Touch-sensitive screens: The technologies and their application. International Journal of Man-Machine Studies, 25, 249-269.

Wechsler, D. (1981). WAIS-R manual. San Antonio, TX: Psychological Corporation. 